\title{
Discordance of physiological and biochemical response to smoking and to psychological stress
}

\author{
CYNTHIA S. POMERLEAU, Ph.D., OVIDE F. POMERLEAU, Ph.D., \\ KATHLEEN McPHEE, M.D. \& ERIC M. MORRELL, Ph.D.
}

Behavioral Medicine Program, University of Michigan, Department of Psychiatry, Riverview Medical Building, 900 Wall Street, Ann Arbor, MI 48105, USA

\begin{abstract}
Both smoking and psychological stress produce marked effects upon cardiovascular function, and several studies have demonstrated that in combination they produce additive or potentiating effects. More recently, it has been reported that individuals strongly reactive to psychological stress are also strongly reactive to nicotine. In an attempt to replicate and extend those findings, we examined reactivity to smoking and competitive mental arithmetic across several physiological and biochemical variables. Despite stable responding across mental arithmetic trials, we were unable to demonstrate significant correlations between reactivity to smoking and to a psychological stressor. We further observed that anxiety level, when low, was a poor predictor of desire to smoke and of withdrawal, whereas higher anxiety levels were more tightly linked to these measures. These findings have implications for the identification of individuals at risk of cardiovascular disease as well as for the design of smoking treatment and relapse prevention programs.
\end{abstract}

\section{Introduction}

MacDougall et al. (1983), using a video game as a laboratory stressor, showed that stress and smoking together produced additive effects upon heart rate in a group of young male subjects. Their findings have been consistently replicated using a variety of psychological stressors for heart rate, though measurements of blood pressure have produced conflicting results (Dembroski et al., 1985; Perkins et al., 1986; Ray et al., 1986; Pomerleau \& Pomerleau, 1987; see Pomerleau \& Pomerleau, 1989 for a more detailed review).

MacDougall et al. (1983) also reported a significant correlation between blood pressure response to a video game alone and to smoking alone. Subsequently, MacDougall et al. (1986) again reported

Correspondence: Cynthia S. Pomerleau, Ph.D. moderately strong correlations between reactivity to stress and reactivity to smoking for systolic and diastolic blood pressure, and speculated that a common mechanism might underlie these phenomena. The absence of comparable correlations for heart rate was interpreted as suggesting that consistency in individual differences in blood pressure was not the result of direct beta-adrenergic influences on the myocardium, but rather was attributable to effects on peripheral resistance that are common to stress and smoking (Trap-Jensen et al., 1979).

The present study was undertaken in order to examine the extent to which reactivity to a psychological stressor is concordant with reactivity to smoking in anticipation of stress across a broader spectrum of physiological and biochemical modalities, including heart rate (HR), systolic and diastolic blood pressure (SBP, DBP), peripheral 
digit temperature (TEMP), and epinephrine and norepinephrine (EPI and NE). State anxiety (ANX), craving or desire to smoke (CRAV) and perception of withdrawal symptoms (WITHD) were also assessed, in order to examine their relationship to one another and to smoking behavior.

\section{Subjects and methods}

Subjects were 26 male smokers recruited from the local community through newspaper advertisements and paid US $\$ 35$ for participating in this study. Only candidates between the ages of 20 and 40 , smoking at least 20 cigarettes per day for at least 5 years, in good health, and free from any drugs or medications that might affect CNS or ANS function, were considered for inclusion. Mean age of subjects was $29.4 \pm 1.1$ (SEM) years, and they had smoked for a mean of $12.7 \pm 1.1$ years. They smoked a mean of $27.8 \pm 1.4$ cigarettes per day, posted a mean score of $6.1 \pm 0.2$ on the Fagerstrom Tolerance Questionnaire (Fagerstrom, 1978), an 8-item assessment of degree of dependence with possible scores ranging from 0 to 11 , and had a mean plasma cotinine level of $284.7 \pm 32.2 \mathrm{ng} / \mathrm{ml}$; these measures indicate a moderate-to-high degree of nicotine dependence (Pomerleau, Fertig \& Shanahan, 1983).

\section{Apparatus}

Subjects sat in an easy chair. The experimenter sat about $1 \mathrm{~m}$ to the subject's right, shielded from his view by a screen. Experimental sequences were controlled via tape-recorded instructions issued through earphones; the earphones also served to muffle environmental noise. A dictaphone was placed on a table next to the subject to permit him to record his answers on the competitive mental arithmetic task.

\section{Laboratory stressor}

The challenge test consisted of 5 minutes of competitive mental arithmetic (subtracting 13's successively from a randomly chosen 4-digit number). Subjects were instructed to continue even if they became confused, since a 'derailment' would count only once as an error and subsequent answers would be accepted as long as they were 13 less than the previous number. Competitive pressure was added by informing subjects that bonuses of US $\$ 50$ and US\$25 would be awarded to the two top scores in each block of 10 subjects based on their combined scores for all trials in the session. (Subjects were not informed in advance as to how many trials would be conducted.) Subjects were taught the task during the original telephone screening and, in an effort to heighten the competitive pressure, were urged to practice ('train') in preparation for the experimental session.

\section{Physiological measures}

HR, SBP and DBP were measured using a Vita-stat model 900 automated blood pressure monitor. TEMP was assessed using J\&J Thermal Monitor model $T-63$, with a sensor taped to the right index finger.

\section{Biochemical measures}

Blood samples for analysis of nicotine, cotinine, EPI and NE were withdrawn unobtrusively from the right median antecubital vein using an $18 \mathrm{G}$ indwelling catheter and $1 \mathrm{~m}$ infusion-exfusion tubing. $\mathrm{A}$ three-way stopcock (Novex: Pharmaseal) was used to fill the tubing with heparinized saline to prevent clotting between samples. Samples were collected in $10 \mathrm{ml}$ sodium EDTA monovettes, after which sodium metabisulfate $(1 \mu \mathrm{g} / \mathrm{ml})$ was added, and chilled in ice water until completion of the session. They were then centrifuged at 2500 r.p.m. for 15 min at $4^{\circ} \mathrm{C}$, pipetted into $1.5 \mathrm{ml}$ aliquot tubes, and stored at $-70^{\circ} \mathrm{C}$. Biochemical analyses were performed by $M$. Hariharan of the University of Michigan Psychiatry Research Assay Support Laboratory (Hariharan, VanNoord \& Greden, 1988).

\section{Assessment of subjective state}

ANX, CRAV and WITHD 'right now' were assessed periodically using Visual Analogue Scales (100 $\mathrm{mm}$ lines), which have been shown for the most part to be psychometrically valid and which were felt to be the most appropriate and least disruptive way of collecting repeated subjective ratings at short intervals (Price $e t$ al., 1983; Wang $e t$ al., 1976). Lines were labeled, respectively, not anxious/extremely anxious, no desire to smoke/extreme desire to smoke and no cigarette withdrawal discomfort/extreme cigarette withdrawal discomfort. Because the ratings were repeated rather than between-group measures, no further instructions 
were offered and subjects remained free to define the terms in accord with their own experience.

\section{Procedure}

Subjects were tested individually. Thirty minutes before the experimental session, subjects were seated in the easy chair and, in order to secure a uniform minimal deprivation period, instructed to smoke a cigarette. The experimental procedure was then explained, after which they were asked to sign an informed consent form. The catheter was inserted in the subject's arm, and subjects listened to music until the beginning of the session. Immediately prior to the start of the session, the earphones were emplaced, and physiological, biochemical and subjective measures were collected.

Because of our desire to avoid confusion of individual differences with order effects in this single-session protocol, all subjects were exposed to the smoking and competitive mental arithmetic in the same standardized sequence. After an adaptation period of about 5 minutes, subjects were asked to smoke one usual-brand cigarette (SMOKE: starting at minute 5). To elicit avid smoking, this interval was followed by a 5 -minute interval of competitive mental arithmetic (MA/S: starting at minute 10). After a recovery period of 35 minutes, subjects were asked to perform a second 5-minute interval of competitive mental arithmetic (MA: starting at minute 50), which served as the index trial for comparison with reactivity to smoking. Physiological, biochemical, and subjective measures were collected every 5 minutes throughout the session, except during a portion of the recovery period (minutes 15-40).

\section{Results}

Table 1 shows the results for all subjective, physiological and biochemical variables collected. (Difficulties in recording prevented collection of accurate skin temperature readings for one subject, who was therefore excluded from all descriptive and inferential analyses of this modality.)

For all physiological and biochemical variables, a one-way repeated measures ANOVA was conducted on the pre and post scores for the SMOKE and MA intervals (i.e. minutes 5, 10,50 and 55). Multiple comparisons using the Dunn-Bonferroni paired $t$-statistics showed that except for TEMP, pre scores (minute 5 versus 50 ) did not differ significantly, indicating that baseline values were recaptured by the time of the MA trial. Both smoking and competitive mental arithmetic were significantly reactive for $H R$ and SBP. TEMP decreased significantly during SMOKE but not during MA; EPI increased significantly during $M A$ but not during SMOKE. DBP and NE were not significantly affected by either SMOKE or MA.

Plasma nicotine values after smoking (minute 10: $\bar{x}=41.3 \pm 2.8 \mathrm{ng} / \mathrm{ml}$ ) were significantly greater than before smoking (minute $5: \bar{x}=23.1 \pm 2.0 \mathrm{ng} / \mathrm{ml}$ ), using a paired $t$-test $(p<0.001)$. Mean nicotine increment was $18.2 \pm 2.4 \mathrm{ng} / \mathrm{ml}$, ranging from a minimum of 1.6 to a maximum of $42.9 \mathrm{ng} / \mathrm{ml}$.

To determine the extent to which the people who were reactive to smoking were the same people who were reactive to mental arithmetic, correlations for HR, SBP, DBP, TEMP, EPI and NE were computed for changes for SMOKE with changes for $M A$. Correlations of changes for MA/S and MA were computed to assess the stability of response to a psychological stressor with and without nicotine from prior smoking. To control for the effects of differences in nicotine intake (Epstein \& Jennings, 1986) and age (Watkins \& Eaker, 1986), and to avoid the problems inherent in correlation of change scores, post-SMOKE values were regressed on preSMOKE values, age and nicotine increment; postMA values were regressed on pre-MA values and age; and post-MA/S values were regressed on postSMOKE values, age and nicotine increment; and appropriate residuals were correlated. As shown in Table 2, no significant correlations were found between smoking and competitive mental arithmetic in any modality. By contrast, significant positive correlations between changes for the two competitive mental arithmetic trials were found in all modalities except for EPI.

In order to examine the extent to which ANX predicted CRAV and WITHD at any given time, Pearson correlation coefficients for these variables were computed. As shown in Table 3, significant correlations were found at minutes 15 (post$\mathrm{MA} / \mathrm{S}$ ), 40, 45, 50 (pre-MA) and 55 (post-MA), but not at minutes 0,5 (pre-SMOKE) and 10 (postSMOKE). Pearson correlations were also computed to determine whether ANX, CRAV or WITHD at minute 5 (pre-SMOKE) predicted subsequent nicotine intake (minute 10-minute 5); non-significant positive correlations of $+0.28,+0.20$ and +0.14 were found for anxiety, desire to smoke and perception of withdrawal symptoms, respectively. No consistent relationships were observed between subjective and physiological variables. 


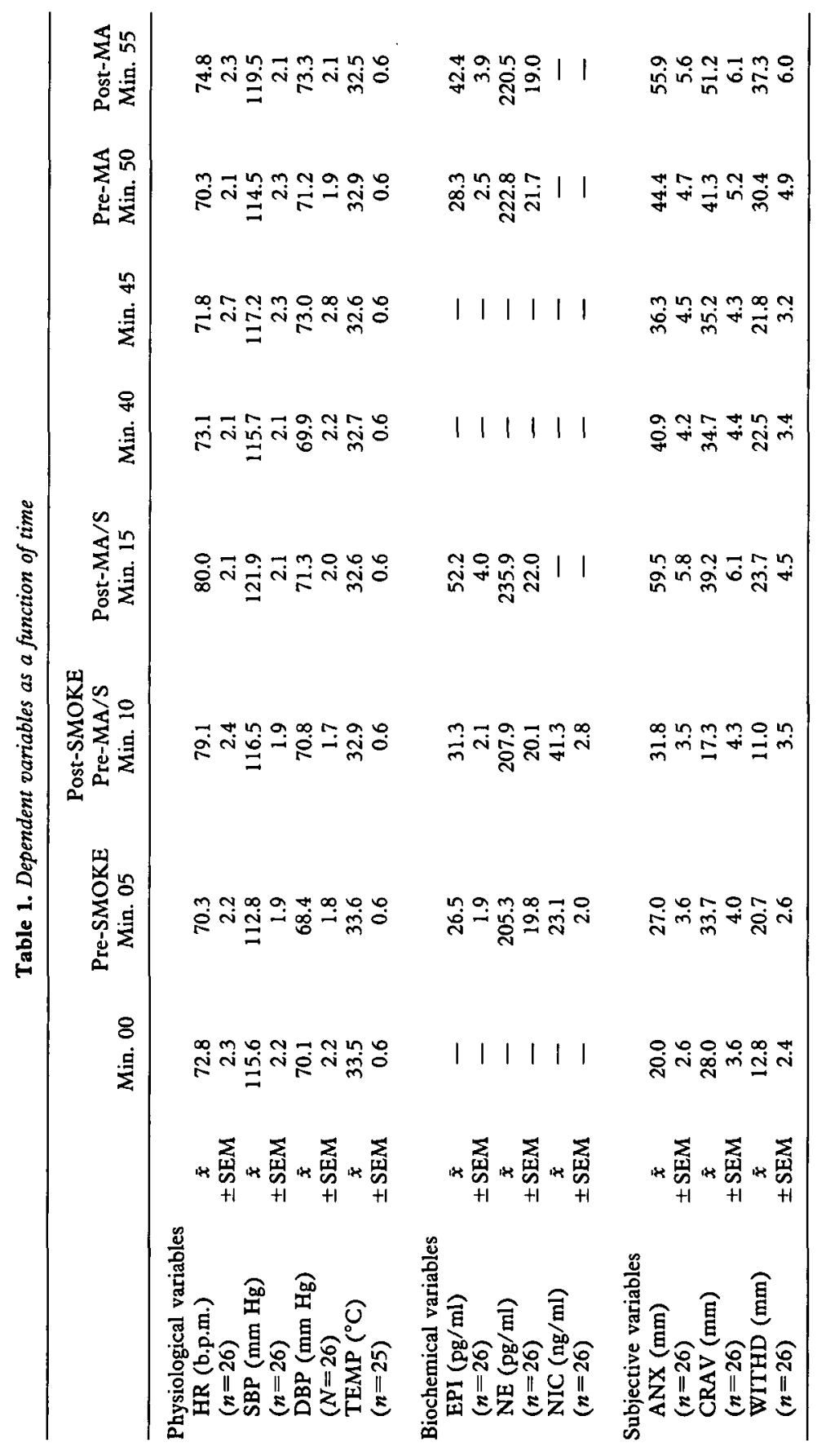


Table 2. Correlation of the effects of smoking (post-SMOKE values regressed on pre-SMOKE values, age and nicotine increment); mental arithmetic (post-MA values regressed on pre-MA values and age); and mental arithmetic preceded by smoking (post-MA/S values regressed on post-SMOKE values, age and nicotine increment)

\begin{tabular}{lccc}
\hline & $\begin{array}{c}\text { Smoking/ } \\
\text { competitive } \\
\text { mental } \\
\text { arithmetic } \\
\text { after sitting } \\
\text { quietly }\end{array}$ & $\begin{array}{c}\text { Smoking/ } \\
\text { competitive } \\
\text { mental } \\
\text { arithmetic } \\
\text { after smoking }\end{array}$ & $\begin{array}{c}\text { Competitive } \\
\text { mental } \\
\text { arithmetic } \\
\text { after sitting } \\
\text { quietly } \\
\text { after smoking }\end{array}$ \\
\hline HR $(n=26)$ & -0.30 & -0.27 & $+0.47^{\star}$ \\
SBP $(n=26)$ & +0.06 & -0.15 & $+0.44^{\star}$ \\
DBP $(n=26)$ & -0.16 & $-0.48^{\star}$ & $+0.43^{\star}$ \\
TEMP $(n=25)$ & +0.28 & +0.03 & $+0.66 \dagger$ \\
EPI $(n=26)$ & +0.15 & +0.11 & +0.33 \\
NE $(n=26)$ & +0.38 & -0.23 & $+0.44^{\star}$ \\
\hline
\end{tabular}

${ }^{\star} p<0.05 ;+p<0.01$.

Table 3. Correlations of desire to smoke and perception of withdrawal symptoms with anxiety at each timepoint

\begin{tabular}{lccc}
\hline Time & $\begin{array}{c}\text { Mean ANX } \\
\text { score (mm) }\end{array}$ & $\begin{array}{c}\text { Correlation } \\
\text { with CRAV }\end{array}$ & $\begin{array}{c}\text { Correlation } \\
\text { with WITHD }\end{array}$ \\
\hline $\begin{array}{l}\text { Minute 0 } \\
\text { Minute 5 } \\
\quad \text { (pre-SMOKE) }\end{array}$ & 20.0 & -0.04 & -0.10 \\
$\begin{array}{c}\text { Minute 10 } \\
\text { (post-SMOKE) }\end{array}$ & 27.0 & +0.26 & +0.31 \\
$\begin{array}{c}\text { Minute 15 } \\
\text { (post-MA/S) }\end{array}$ & 31.8 & +0.24 & +0.14 \\
$\begin{array}{l}\text { Minute 40 } \\
\text { Minute 45 }\end{array}$ & 59.5 & $+0.45^{\star}$ & $+0.49^{\star}$ \\
$\begin{array}{c}\text { Minute 50 } \\
\text { (pre-MA) }\end{array}$ & 40.9 & $+0.52 \dagger$ & $+0.48^{\star}$ \\
$\begin{array}{c}\text { Minute 55 } \\
\text { (post-MA) }\end{array}$ & 36.3 & $+0.47^{\star}$ & $+0.58 \dagger$ \\
\hline
\end{tabular}

${ }^{\star} p<0.05 ; \dagger p<0.01 ; \ddagger p<0.001$.

\section{Discussion}

Both smoking and competitive mental arithmetic caused significant increases in HR and SBP. In addition, significant decreases in TEMP were observed after smoking and significant increases in EPI after competitive mental arithmetic. Thus, as expected, both of our manipulations were effective in producing marked changes in several measures of sympathetic reactivity. The mental arithmetic interval following smoking (MA/S, minutes $10-15$ ) was not entered into the analysis because the design does not permit teasing out whether the effects were attributable to the first presentation of the stressor, . to the persistent effects of nicotine or to both.
Nonetheless, the sharp increases in catecholamines seen at minute 15 suggest that further investigation of these variables in a stress/smoking paradigm may be fruitful.

We found no evidence to support the idea that subjects who were highly reactive to smoking were also highly reactive to competitive mental arithmetic in any modality. Our confidence in the reliability of the present findings is strengthened by the stability of response to competitive mental arithmetic on the two trials, which shines through despite individual differences in nicotine level and possible ceiling effects on the first trial, as well as possible attenuation of response due to habituation 
on the second trial. In fact, the lack of a strong correlation between reactivity to smoking and to a psychological stressor should perhaps not be surprising, since even if there are shared underlying mechanisms, chronic tolerance to the effects of nicotine might be expected to have an impact on reactivity to smoking but not necessarily to psychological stress. Although chronic tolerance to nicotine has not been systematically studied in humans (USDHHS, 1988), the phenomenon has been demonstrated for HR (Perkins et al., 1989; Pomerleau et al., 1983) and may extend to other modalities as well.

Our findings are in sharp contrast to those of MacDougall et al. (1986), who found very strong correlations that were stable over a period of around 7 weeks; since our study had exactly the same number of subjects, our sample size should also have been adequate to detect such effects, even allowing for statistical shrinkage. Several methodological differences may have contributed to these discrepancies: (1) Their method of computing difference scores using the lowest baseline values collected rather than the value immediately preceding the manipulation exaggerated their correlations, as they themselves observed. (2) We controlled for smokers' ability to regulate nicotine intake statistically, by partialing plasma nicotine boost out of physiological changes during smoking and the subsequent mental arithmetic trial. MacDougall et al. attempted to control experimentally, by using a paced smoking procedure and making special efforts to keep subjects relaxed while smoking; this procedure may have helped to reduce individual variability in nicotine intake, though in the absence of measures of plasma nicotine this supposition cannot be substantiated, and findings in our own laboratory suggest that paced puffing is not an effective method of achieving fixed dosing (Pomerleau, Majchrzak \& Pomerleau, 1989). (3) MacDougall et al. do not specify the age of their subjects; possibly they were younger and more uniform with respect to nicotine tolerance than ours. (4) Their collection of repetitive measures for each variable may have produced more stable, 'smoother' results; and collection of measures during rather than following the mental arithmetic trials (which would more nearly have approximated peak responses and avoided the possibility of 'relief' at the termination of the stressor) may have produced more truly representative indices of cardiovascular reactivity.

The puzzling inconsistencies between these two studies cannot be fully resolved without more extensive research involving a validated method of delivering controlled doses of nicotine (see Pomerleau, Pomerleau \& Rose, 1989b) and application of parametric doses of nicotine in combination with different classes and intensities of stressors, with order of presentation varied to confirm that all findings are fully generalizable. Both our study and that of MacDougall et al. involved a naturalistic deprivation time of 30 minutes; in view of the development of acute tolerance (Porchet et al., 1989; West \& Russell, 1987), a 'cleaner' measure of cardiovascular reactivity would be gained by testing subjects after overnight abstinence. Subject factors such as chronic tolerance to nicotine, age and gender must also be investigated systematically. Until this agenda has been accomplished, the extent to which reactivity to smoking it concordant with reactivity to psychological stress must remain an open question.

The resolution of this issue has implications for the identification and treatment of individuals at risk for cardiovascular disease. Cigarette smoking has been definitively established as a risk factor for coronary heart disease (USDHHS, 1983), and the suggestion has also been advanced that exaggerated cardiovascular responses to stressors may have cumulative detrimental effects upon the cardiovascular system (Krantz et al., 1988). If indeed the people who are highly reactive to nicotine are not necessarily highly reactive to psychological stressors, then the individuals at greatest risk may be those who exhibit a dual diathesis, a predisposition towards reactivity both to smoking and to psychological stressors. Future research concentrating upon such probands, using both laboratory challenges and longitudinal studies, may be of value in achieving an accurate evaluation of these two risk factors in combination.

Correlations of ANX with CRAV and WITHD were relatively weak at minutes 0 and 5 (preSMOKE), when mean anxiety level was also low, and at minute 10 , immediately after smoking, but considerably stronger at the higher levels of anxiety recorded at minutes 15 (post-MA/S), 40, 45, 50 (pre-MA) and 55 (post-MA). Although our results suggest that anxiety level is a poor predictor of likelihood of smoking at low anxiety levels but a much better predictor at higher anxiety levels, several caveats must be stated. First, the failure to find stronger correlations of anxiety with desire to smoke and withdrawal at lower levels may simply be a function of range restriction (as indicated by the relatively low variability at these levels). Second, a 
longer deprivation period might have produced ratings that were more meaningful and less subject to demand characteristics. Finally, since we did not pre-establish the definition of 'anxiety' or 'withdrawal discomfort' with our subjects, correlations between these variables may be an artifact of subjects' confusing the two-a possibility that may help to explain the secular trend toward an increase in anxiety ratings over the course of the session, though it is not supported by ratings immediately after smoking (minute 10), when mean withdrawal decreased but mean anxiety ratings rose.

Although the failure of any of the subjective measures to predict subsequent nicotine intake as measured by changes in plasma nicotine is troubling, a better test of this question would require a smoking trial in a nonstressful context to control for individual differences in characteristic levels of nicotine intake. Moreover, such variables may have a more robust effect upon frequency of smoking than upon nicotine intake during the smoking of a single cigarette. (In this connection, it is of interest to observe that self-reported desire to smoke and perception of withdrawal discomfort were as high at minute 15 , right after mental arithmetic but only 5 minutes after smoking, as they were 25 minutes later, even though no smoking had occurred in the interim.) Research on latency to smoking under varying conditions of anxiety may shed further light on the possibility that smoking is likely to occur sooner at higher anxiety levels.

The possible anxiolytic effects of smoking have been the subject of much discussion, with mixed findings reported in the literature (see Jarvik et al., 1989). Because of the possible confounding effects of sequence, it is not possible to evaluate the direct effects of smoking on anxiety in our study. Certainly smoking did not cause a decrease in anxiety during the subsequent mental arithmetic trial; on the other hand, it appears that the increase in anxiety during the smoking interval itself (minute 10-minute 5) is smaller than the increase in the comparable 5 minutes preceding the second mental arithmetic trial (minute 50-minute 45). Regardless of the actual impact of smoking on anxiety, however, our finding that high anxiety levels may be associated with desire to smoke and perception of withdrawal symptomatology supports the contention that the relationship between psychological stress and smoking needs to be addressed vigorously in the context of smoking cessation and relapse prevention programs (Abrams et al., 1987).

\section{Acknowledgements}

This research was supported by University of Michigan Psychiatry Research Committee funding to the first and fourth authors and by National Cancer Institute grant CA/DA 42730 to the second author.

\section{References}

Abrams, D. A., Monti, P. M., Pinto, R. P., Elder, J. P., Brown, R. A. \& Jacobus, S. I. (1987) Psychosocial stress and coping in smokers who relapse or quit, Health Psychology, 6, pp. 289-303.

Dembroski, T. M., MacDougall, J. M., Cardozo, S. R., Ireland, S. K. \& KRUG-FITE, J. (1985) Selective cardiovascular effects of stress and cigarette smoking in young women, Health Psychology, 4, pp. 153-167.

EPSTEIN, L. H. \& JeNNINGS, J. R. (1986) Smoking, stress, cardiovascular reactivity, and coronary heart disease, in: Matthews, K. A., Weiss, S. M., Detre, T., DemBROSKI, T. M., FALKNER, B., MANUCK, S. B. \& WILliams, R. B., JR (Eds) Handbook of Stress, Reactivity $\mathcal{E}$ Cardiovascular Disease, pp. 291-309 (New York, John Wiley \& Sons).

FAGERSTROM, K. (1978) Measuring degree of physical dependence to tobacco smoking with reference to individualization of treatment, Addictive Behaviors, 3, pp. 235-241.

Hariharan, M., VanNoord, T. \& Greden, J. F. (1988) A high-performance liquid-chromatographic method for routine simultaneous determination of nicotine and cotinine in plasma, Clinical Chemistry, 34, pp. 724-729.

Jarvik, M. E., Caskey, N. H., Rose, J. E., Herskovic, J. E. \& SADEghpour, M. (1989) Anxiolytic effects of smoking associated with four stressors, Addictive Behaviors, 14, pp. 379-386.

Krantz, D. S., Contrada, R. J., Hill, D. R. \& FrIEDLER, E. (1988) Environmental stress and biobehavioral antecedents of coronary heart disease, fournal of Consulting and Clinical Psychology, 56, pp. 333-341.

MacDougall, J. M., Dembroski, T. M., SlaAts, S., HeRD, J. A. \& ELIOT, R. S. (1983) Selective cardiovascular effects of stress and cigarette smoking, fournal of Human Stress, 9, pp. 13-21.

MacDougall, J. M., Musante, L., Howard, J. A., Hanes, R. L. \& Dembroski, T. M. (1986) Individual differences in cardiovascular reactions to stress and cigarette smoking, Health Psychology, 5, pp. 531-544.

Perkins, K. A., Epstein, L. H., Jennings, J. R. \& STILlER, R. (1986) The cardiovascular effects of nicotine stress, Psychopharmacology, 90, pp. 373-378.

Perkins, K. A., Epstein, L. H., Stiller, R. L., Marks, B. L. \& JACOB, R. G. (1989) Chronic and acute tolerance to the heart rate effects of nicotine, Psychopharmacology, 97(4), pp. 529-534.

Price, D. D., McGrath, P. A., Rafil, A. \& BuckingHAM, B. (1983) The validation of visual analog scales as ratio scale measures for chronic and experimental pain. Pain, 17, pp. 45-56.

Pomerleau, C. S. \& Pomerleau, O. F. (1987) The effects of a psychological stressor on cigarette smoking and subsequent behavioral and physiological responses, Psychophysiology, 24, pp. 278-285. 
Pomerleau, O. F. \& Pomerleau, C. S. (1989) Stress, smoking, and the cardiovascular system, fournal of Substance Abuse, 1, pp. 331-343.

Pomerleau, O. F., Fertig, J. \& Shanahan, S. O. (1983) Nicotine dependence in cigarette smoking: an empirically-based, multivariate model, Pharmacology Biochemistry \&o Behavior, 19, pp. 291-299.

Pomerleau, C. S., Majchrzak, M. J. \& Pomerleau, O. F. (1989a) Paced puffing as a method for administering fixed doses of nicotine, Addictive Behaviors, 14, pp. 571-575.

Pomerleau, O. F., Pomerleat, C. S. \& Rose, J. E. (1989b) Controlled dosing of nicotine: a review of problems and progress, Annals of Behavioral Medicine, 11, pp. 158-163.

Porchet, H. C., Benowitz, N. L., Sheiner, L. B. \& COPELAND, J. R. (1989) Apparent tolerance to the acute effect of nicotine results in part from distribution kinetics, fournal of Clinical Investigation, 80, pp. 1466-1471.

RAY, R. L., Nellis, M. J., BRAdy, J. V. \& Foltin, R. W. (1986) Nicotine and caffeine effects on the task-elicited blood pressure response, Addictive Behaviors, 11, pp. 31-36.
Trap-Jensen, J., Carlsen, J. E., Svendsen, T. L. \& Christensen, N. J. (1979) Cardiovascular and adrenergic effects of cigarette smoking during immediate nonselective and selective beta-adrenergic blockade in humans, European Yournal of Clinical Investigation, 9, pp. 181-183.

US DePartment OF Health AND Human SERVICE (1988) The health consequences of smoking: nicotine addiction, Publication DHHS [CDC] 88-84-06 (Washington, DC, US Government Printing Office).

WANG, R. I. H., WIESEN, R. L., TReul, S. \& STOCKDALE, S. (1976) A brief anxiety rating scale in evaluating anxiolytics, fournal of Clinical Pharmacology, 26, pp. 99-105.

WATKINS, L. O. \& EAKER, E. (1986) Population and demographic influences on reactivity, in: MATTHEws, K. A., Weiss, S. M., Detre, T., Dembroski, T. M., Falkner, B., ManuCK, S. B. \& Williams, JR, R. B. (Eds) Handbook of Stress, Reactivity $\mathcal{E}$ Cardiovascular Disease, pp. 291-309 (New York, John Wiley \& Sons).

WEST, R. J. \& Russell, M. A. H. (1987) Cardiovascular and subjective effects of smoking before and after $24 \mathrm{~h}$ of abstinence from cigarettes, Psychopharmacology, 92, pp. 118-121. 
This document is a scanned copy of a printed document. No warranty is given about the accuracy of the copy. Users should refer to the original published version of the material. 